\title{
On Surface Losses in Direct Metal Laser Sintering Printed Millimeter and Submillimeter Waveguides
}

\author{
Max Holmberg ${ }^{1}$ - Dragos Dancila ${ }^{1} \cdot$ Anders Rydberg $^{1}$ (D) . \\ Björgvin Hjörvarsson ${ }^{2}$ - Ulf Jansson ${ }^{3}$ - Jithin James Marattukalam² . \\ Niklas Johansson ${ }^{2}$ Joakim Andersson ${ }^{1}$
}

Received: 9 October 2017 / Accepted: 31 January 2018 /

Published online: 21 February 2018

(C) The Author(s) 2018. This article is an open access publication

\begin{abstract}
Different lengths of WR3 (220-330 GHz) and WR10 (75-110 GHz) waveguides are fabricated through direct metal laser sintering (DMLS). The losses in these waveguides are measured and modelled using the Huray surface roughness model. The losses in WR3 are around $0.3 \mathrm{~dB} / \mathrm{mm}$ and in WR10 $0.05 \mathrm{~dB} / \mathrm{mm}$. The Huray equation model is accounting relatively good for the attenuation in the WR10 waveguide but deviates more in the WR3 waveguide. The model is compared to finite element simulations of the losses assuming an approximate surface structure similar to the resulting one from the DMLS process.
\end{abstract}

Keywords Millimeterwave $\cdot 3 D$-metal-printed $\cdot$ Waveguide loss

\section{Introduction}

Passive millimetre-wave and $\mathrm{THz}$ components are traditionally fabricated by computer numerical control (CNC), micromachining, electrical discharge machining (EDM), or injection molding. 3D-printing is a fairly new fabrication technique and involves

Anders Rydberg

anders.rydberg@angstrom.uu.se

1 Department of Engineering Sciences, Angstrom Laboratory, Uppsala University, Lägerhyddsvägen 1, Uppsala 752 37, Sweden

2 Department of Physics and Astronomy, Angstrom Laboratory, Uppsala University, Lägerhyddsvägen 1, Uppsala 752 37, Sweden

3 Department of Chemistry, Angstrom Laboratory, Uppsala University, Lägerhyddsvägen 1, Uppsala 752 37, Sweden 
technologies, such as fused deposition modelling (FDM), stereolithography apparatus (SLA), ceramic stereolithography apparatus (CSLA), microstereolithography apparatus ( $\mu \mathrm{SLA})$, binder jetting $(\mathrm{BJ})$, polymer jetting $(\mathrm{PJ})$, selective laser melting (SLM), selective laser sintering (SLS), and electron beam melting (EBM). Several comprehensive reviews of the different technologies can be found in the literature (see, e.g. ref. [1]) and will not be discussed in this paper. Some of these techniques have been used for fabrication of $\mathrm{THz}$ devices with an emphasis on optic components fabricated using, e.g. multi-jet-modelling in polylactide for creating optics or in 3D printed polymer resin which is sputter deposited with $\mathrm{Au}$ [2, 3]. 3D-metal printing by selective laser melting (SLM) was used for the fabrication of terahertz optics in the form of a zone plate operating at $530 \mathrm{GHz}$, with over $90 \%$ reflection efficiency [4]. Waveguides have been fabricated for the WR-90 and WR-10 waveguide bands using fused deposition modelling (FDM) and stereolithography apparatus (SLA) respectively [5]. The fabricated WR10 copper waveguide showed an attenuation of $11 \mathrm{~dB} / \mathrm{m}$ which is comparable to commercial machined aluminium waveguides [5]. In this paper, we investigate the surface loss mechanism theoretically and experimentally in WR3 and WR10 stainless steel waveguides fabricated using direct metal laser sintering (DMLS). Waveguides have previously been fabricated with the help of DMLS for the WR10 frequency band see, e.g. ref. [6] where a 90-degree twist for the WR10 was successfully fabricated. Horn antennas have also been fabricated with metal 3D-printing technology [7] performing well in comparison with commercial horn antennas.

In this paper, different lengths of WR3 and WR10 waveguides are fabricated with DMLS, see Fig. 1. The losses of these waveguides are examined by measurements and compared to analytical calculations and HFSS simulations [8]. The Huray surface roughness model applicability to waveguides in the WR3 and WR10 bands is investigated. The fine waveguide surface topology is investigated with a scanning electron microscope (SEM). An approximate surface roughness model structure is implemented in Ansoft HFSS.

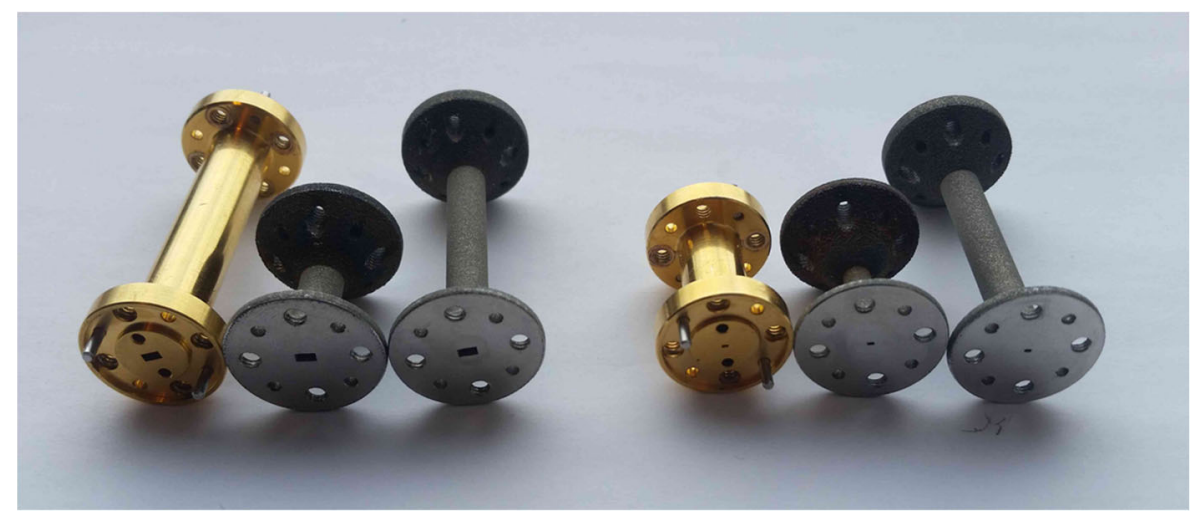

Fig. 1 The waveguides that were examined. To the left are the WR10 waveguides and to the right are the WR3. The golden waveguides are commercial [9] surface gold plated waveguides used for reference 


\section{Fabrication}

The waveguides are fabricated in a DMLS process using EOS M100 SLM system with gas atomized Stainless Steel 316L Surface 1.0 powder, containing particles of size distribution between 20-50 $\mathrm{\mu m}$. Standard 316L SS process parameters developed by EOS $\mathrm{GmbH}$ is utilized for the $3 \mathrm{D}$ printing of the waveguides. A 3D CAD model of the parts to be built is generated using the SolidWorks software. The 3D CAD model is numerically sliced into a number of layers in cross section along the direction of build with the help of the slicer software, EOS PRINT. These sliced layers are sequentially recreated on a build plate, which acts as a substrate to produce the $3 \mathrm{D}$ printed waveguides. The layer deposition thickness is always maintained at $20 \mu \mathrm{m}$ and the components are manufactured under controlled atmosphere with Argon as the process gas. The lengths of the fabricated waveguides are 25 and $50 \mathrm{~mm}$ for the smaller and larger waveguides respectively.

\section{Theory}

There are a number of different models for calculating the attenuation in a conductor due to surface roughness. The simplest is the Morgan-Hammerstad [10]; however, this model perform poorly for frequencies above $4 \mathrm{GHz}$ [11]. A modified version of the Morgan-Hammerstad model is the Groisse equation which perform better at slightly higher frequencies (up to around $12 \mathrm{GHz}$ ) [11]. However, for the WR3 (220$330 \mathrm{GHz})$ and WR10 $(75-110 \mathrm{GHz})$ bands, these models are inadequate since they produce large errors at these high frequencies. Furthermore, the Morgan-Hammerstad and Groisse models are based on equations that assume the attenuation due to surface roughness in a conductor not to be higher than a factor of two compared to a perfectly smooth conductor. Thus, in cases of high wall roughness, the calculated results are incorrect [12].

Another model is the Huray Model [13] which have been proven to model the attenuation due to surface roughness accurately up to $50 \mathrm{GHz}$ and predicted to work accurately up to $100 \mathrm{GHz}$. The Huray model needs a statistical distribution of sphere radii as input; however, if one simplifies the model to include only a single radius size the following equation is obtained (see also Section 5 Discussion),

$$
\frac{P_{\text {rough }}}{P_{\text {smooth }}} \approx 1+\frac{3 S R}{2\left(1+\frac{\delta(f)}{a}+\frac{\delta(f)^{2}}{2 a^{2}}\right)}
$$

The relation $\frac{\text { Prough }}{\text { Psmooth }}$ is the ratio of power loss caused by a rough conductor versus a perfectly flat conductor, $\delta$ is the skin depth of the material (see Eq. 3), $a$ is the radius of the spheres and SR is the Hall-Huray surface ratio defined by,

$$
S R=\frac{4 \pi N a^{2}}{A_{\text {flat }}}
$$


where $N$ is the number of spheres on an area $A_{\text {flat }}$. The following equation describes the skin depth, $\delta[14]$,

$$
\delta=\sqrt{\frac{2 \rho}{\omega \mu}} \sqrt{\sqrt{1+(\rho \omega \epsilon)^{2}}+\rho \omega \epsilon}
$$

where $\rho$ is the resistivity, $\mu$ is the permeability, $\omega$ the angular frequency, and $\epsilon$ the permittivity.

\section{Measurements and Simulations}

The S-parameter measurements are performed using a vector network analyser Keysight N5225A PNA [15] with WR3 and WR10 mm-wave extenders from OML Inc [9]. The mm-extenders consist of one T/R-extender and one T-extender creating a one-path two ports measurement setup. In Fig. 2, the schematic of the mm-wave extenders can be seen [16]. The VNA was calibrated using an enhanced response calibration, with a calibration kit consisting of terminations in the form of open, load and short. Also, a thorough measurement was part of the calibration. The measurements of the waveguides were performed by connecting the waveguide in-between the mm-wave extenders and measuring the scattering parameters S11 and S21. RF and LO input powers were $10 \mathrm{dBm}$.

The transmission coefficient in the waveguides was measured and a mean value with regards to the waveguide lengths was calculated. In Fig. 3, the transmission coefficients of the WR10 gold plated reference and 3D-printed waveguide are shown. The transmission coefficient of the reference waveguide is around $0.005 \mathrm{~dB} / \mathrm{mm}$ and the $3 \mathrm{D}$-printed waveguide is about $0.06 \mathrm{~dB} / \mathrm{mm}$. For the WR3 waveguides, as can be seen in Fig. 4, the measured reference waveguide (gold plated) is about $0.05 \mathrm{~dB} / \mathrm{mm}$ and the $3 \mathrm{D}$-printed steel waveguide is about $0.3 \mathrm{~dB} / \mathrm{mm}$.

There are different sources for the attenuation: (i) the difference in surface material (steel versus gold plating), (ii) surface roughness and (iii) difference in interior dimension of the waveguide due to the 3D-printing. In Fig. 5a, b, one can see that the waveguide entrance is not a perfect rectangle and that the surface roughness is high, both these factors will affect the attenuation.
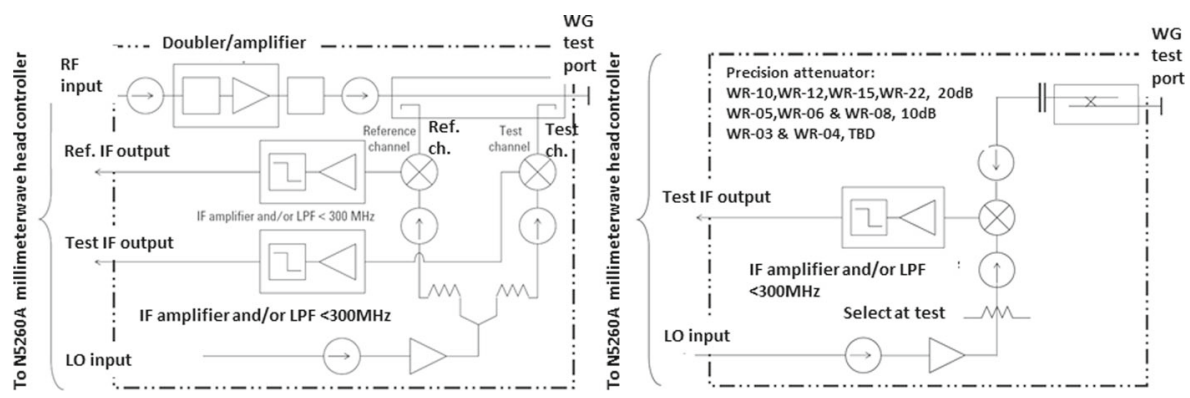

Fig. 2 Schematic drawing of the $T / R$ (to the left) and the $T$ (to the right) modules from OML inc. that were used in the measurements. For better resolution of the figure see ref. [16] 


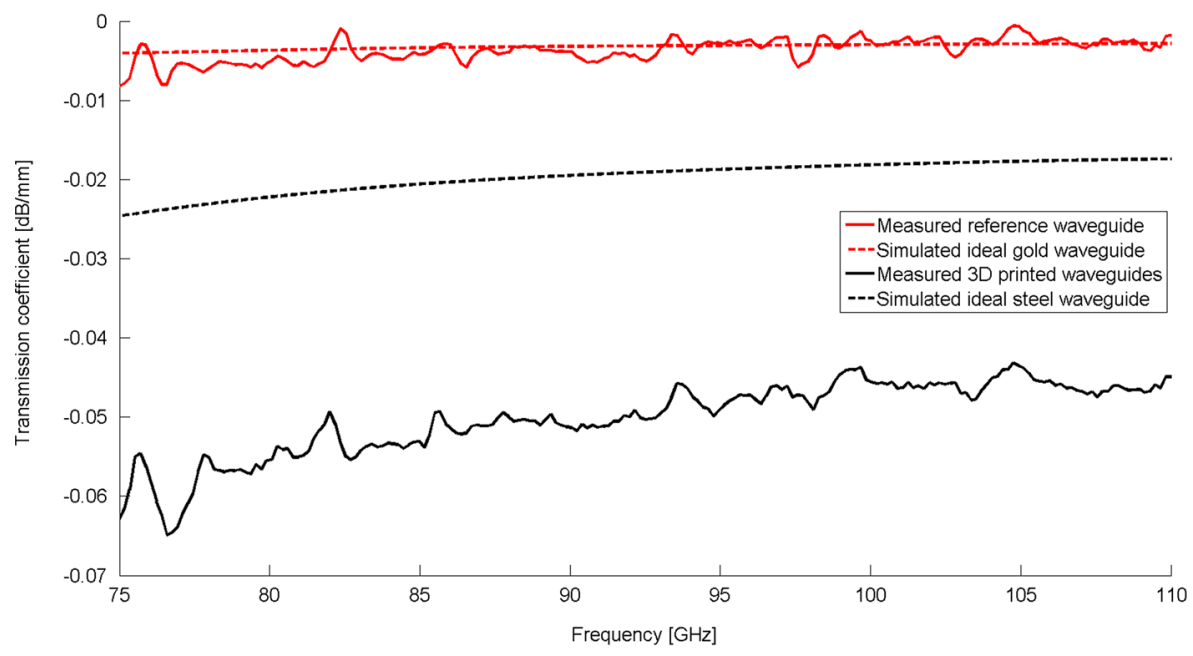

Fig. 3 Transmission coefficient as a function of frequency for the WR10 waveguide. Comparison of reference and 3D-printed waveguide. Solid red line: measured reference gold waveguide. Dashed red line: simulated ideal gold waveguide. Solid black line: measured 3D-printed waveguide. Dashed black line: simulated ideal steel waveguide

In order to analyse the sources of attenuation, the waveguide surfaces are inspected with the help of a Veeco Wyko NT1100 optical interferometry measurement system and a Scanning Electron Microscope (SEM), see Figs. 6 and 5b, respectively.

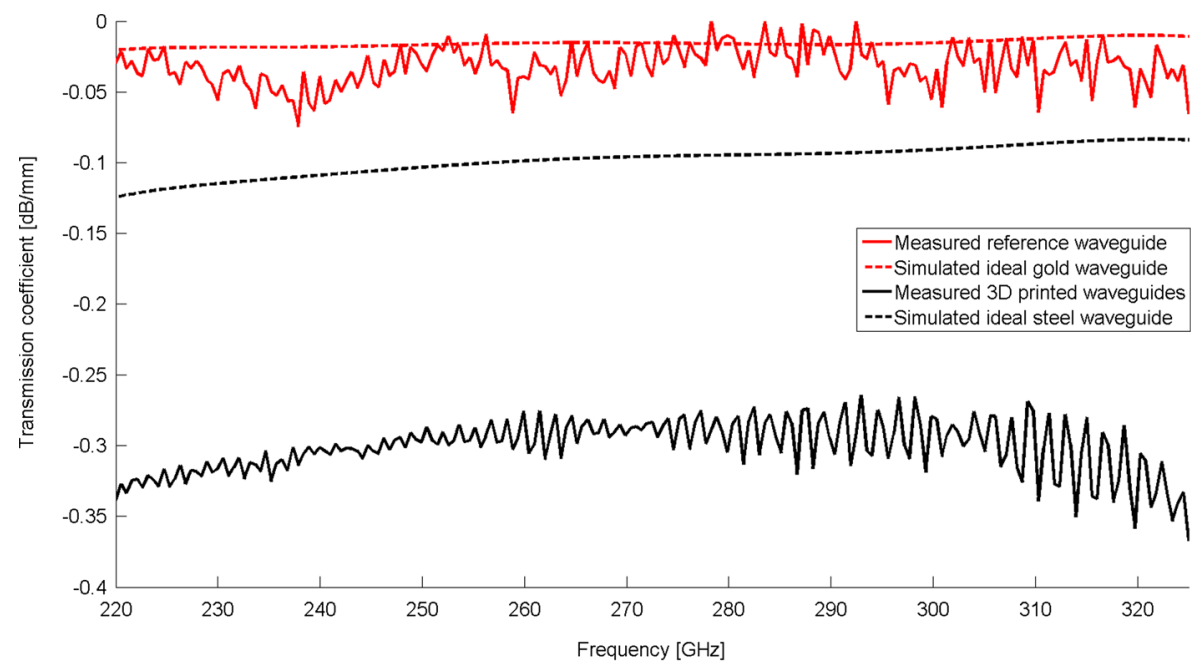

Fig. 4 Transmission coefficient as a function of frequency for the WR3 waveguide. Comparison of reference and 3D-printed waveguide. Solid red line: measured reference gold waveguide. Dashed red line: simulated ideal gold waveguide. Solid black line: measured 3D printed waveguide. Dashed black line: simulated ideal steel waveguide 

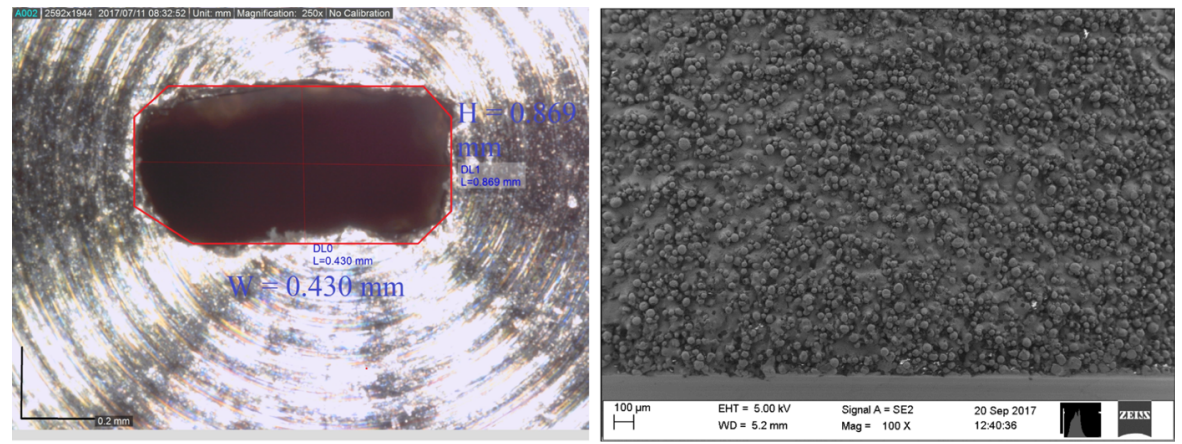

(a) A zoomed in view from the end of the stainless steel (b) SEM image of the surface structure in the stainless steel WR3 waveguide. A sketch of roughly how the geometrical waveguides. HFSS model with chamfered corners looks like can be seen in the thick red contour of the waveguide hole.

Fig. 5 Microscope pictures of the waveguides

The levelled cross-section (see Fig. 6b) obtained from the Veeco optical interferometry measurements in the investigated area of $90 \mu m \times 120 \mu m$ (see Fig. 6a) shows the absolute heights of red $(+58 \mu \mathrm{m})$ and green islands $(+48 \mu \mathrm{m})$ while the ground floor is at $(+29 \mu \mathrm{m})$ resulting in the relative heights of about $+30 \mu \mathrm{m}$ for the red and $+20 \mu \mathrm{m}$ for the green islands.

(a)

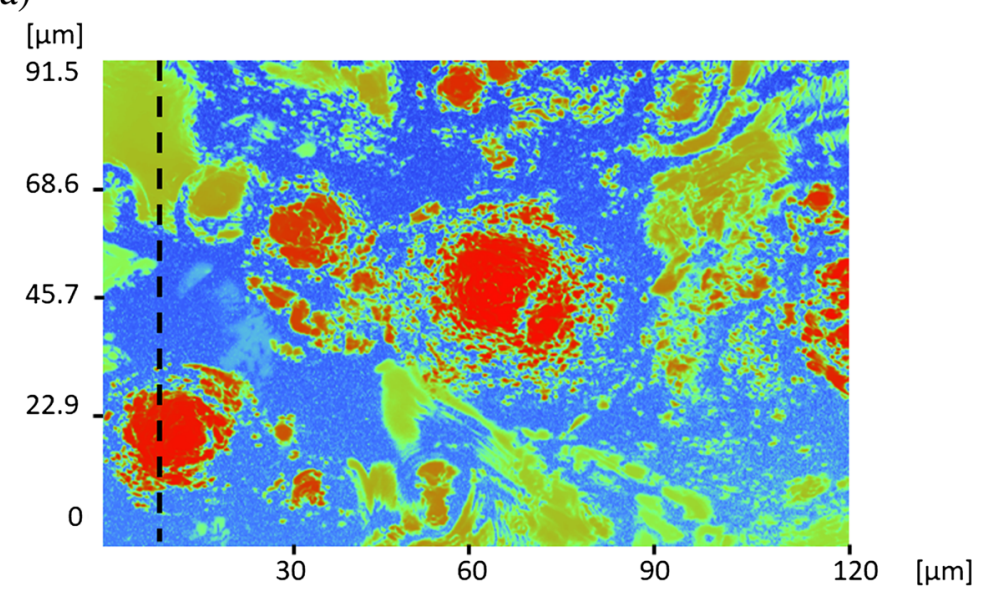

(b)

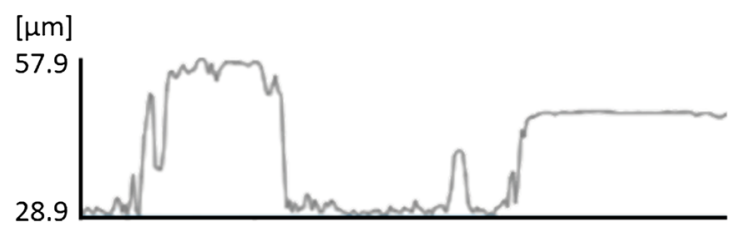

Fig. 6 Export from the Veeco optical interferometry measurements showing a the surface roughness distribution on the investigated area of $90 \mu \mathrm{m} \times 120 \mu \mathrm{m}$ and $\mathbf{b}$ the levelled cross-section at horizontal coordinate $=10 \mu m(\operatorname{see}(\mathbf{a}))$ 
There is a gradual transition between the points at the ground level and the $+20 \mu \mathrm{m}$ level, arising from the green islands and the red islands reaching at the level $+30 \mu \mathrm{m}$. The greenish islands consists of half melted steel particles of height $+20 \mu \mathrm{m}$ (see Fig. 6b) and the red areas are the particles resting as islands of height $+30 \mu \mathrm{m}$ (see Fig. 6b).

The Huray model is based on evaluating the surface area of a number of spherical particles over a specific area. The number of spheres with a diameter of $20 \mu \mathrm{m}$ in an area of $100 \mu m \times 100 \mu m$ (see Fig. 5b) ranges from between 2 and 5 with an average of 3 spheres.

From this, the equivalent surface area of the roughness in the investigated area is evaluated considering the height distribution and the levelled cross-section in Fig. 6. To evaluate the equivalent roughness surface area, significant particles are approximated by right rectangular prisms, considering the relatively flat top at different heights. The equivalent roughness surface area is the summation of the top and side walls of these rectangular prisms, randomly distributed in the investigated area.

The evaluated surface ratio (see Eq. 2) is about 1.1 which will be used in the analytical calculations. This SR-value along with the particle radius of $10 \mu \mathrm{m}$ is used in the simulation and calculations of $P_{\text {rough }}(1)$. Included in the simulation are also the chamfered corners of the 3D-printed waveguide (see Fig. 5a).

The reflection losses were found to be $16.2 \mathrm{~dB}$ for the WR10 and $19.8 \mathrm{~dB}$ for the WR3 at the centre of the waveguide bands. The inclusion of these values in the calculation of the attenuation (see Eq. 2 in ref. [5]) showed only a very small change in the metal and scattering losses and thus the reflection losses are considered negligible.

A comparison between a simulated ideal steel waveguide and the 3D-printed waveguides is shown in Figs. 3 and 4, giving a better understanding of this source of losses. One can see as expected that the choice of material has a large influence on the losses in the waveguides. With an assumed ideal steel waveguide, the difference between the steel waveguide and the 3D-printed one becomes on average $0.029 \mathrm{~dB} / \mathrm{mm}$ for WR10 and $0.18 \mathrm{~dB} / \mathrm{mm}$ for WR3. Thus, using gold as the surface material will as expected make an improvement (see Section 5) but is not the major cause for the losses, as can be seen.

Suggestions were put forward by the reviewers of this paper to compare the results for the fabricated WR10 waveguide with the WR10 waveguide fabricated in ref. [5]. Extrapolation of the measurements, results in an attenuation of about $7 \mathrm{~dB} / \mathrm{m}$ assuming a conductivity of $5.96 * 10^{7} \mathrm{~S} / \mathrm{m}$ for copper material compared to the conductivity of $1.45 * 10^{6}$ for the steel material used. This value includes then also the losses due to the surface roughness (calculated with the Huray model) in a copper waveguide created with the DMLS process. Thus, the DMLS would in this relatively simple comparison, give a better or comparable performance compared to the fabricated WR10 copper waveguide in ref. [5] showing an attenuation of $11 \mathrm{~dB} / \mathrm{m}$. It should also in this comparison be taken into account that different fabrication materials can also by themselves give different surface roughness due to differences in the melting process, experienced by the authors.

The results after implementing the Huray-model are shown in Figs. 7 and 8, using a radius (a) of $10 \mu \mathrm{m}$ for the particles. As can be seen for both the WR10 and WR3 graphs the Huray-model matches the measured values fairly well, slightly better for 


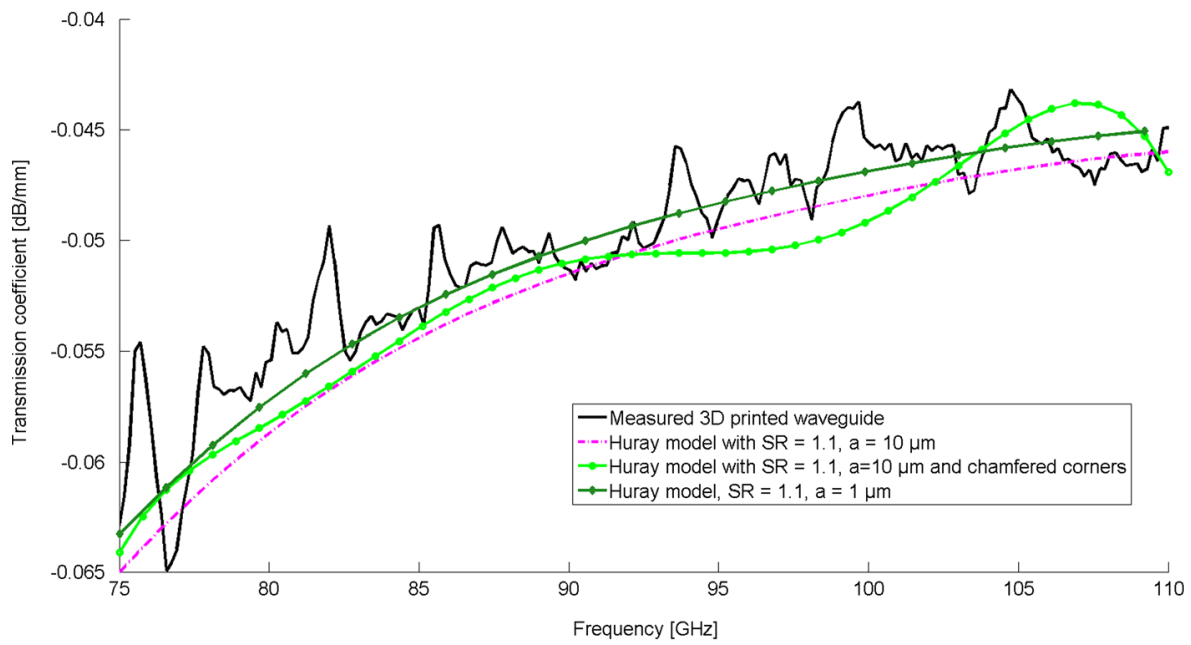

Fig. 7 Transmission coefficient as a function of frequency for the WR10 waveguide. Comparison of measured 3D-printed waveguide and Huray model. Solid black line: measured 3D printed waveguide. Pink: Huray model with $\mathrm{SR}=1.1, \mathrm{a}=10 \mu \mathrm{m}$. Bright green (more dots along curve): Huray model with $\mathrm{SR}=1.1, \mathrm{a}=10 \mu \mathrm{m}$ and chamfered corners. Dark green (fewer dots along curve): Huray model, $\mathrm{SR}=$ $1.1, \mathrm{a}=1 \mu \mathrm{m}$

the WR10-waveguide. For the WR10 waveguide, we see a small difference between the simulated results for the waveguide with chamfered corners and a normal waveguide with 90-degree corners. However, for the WR3-waveguide, the difference is

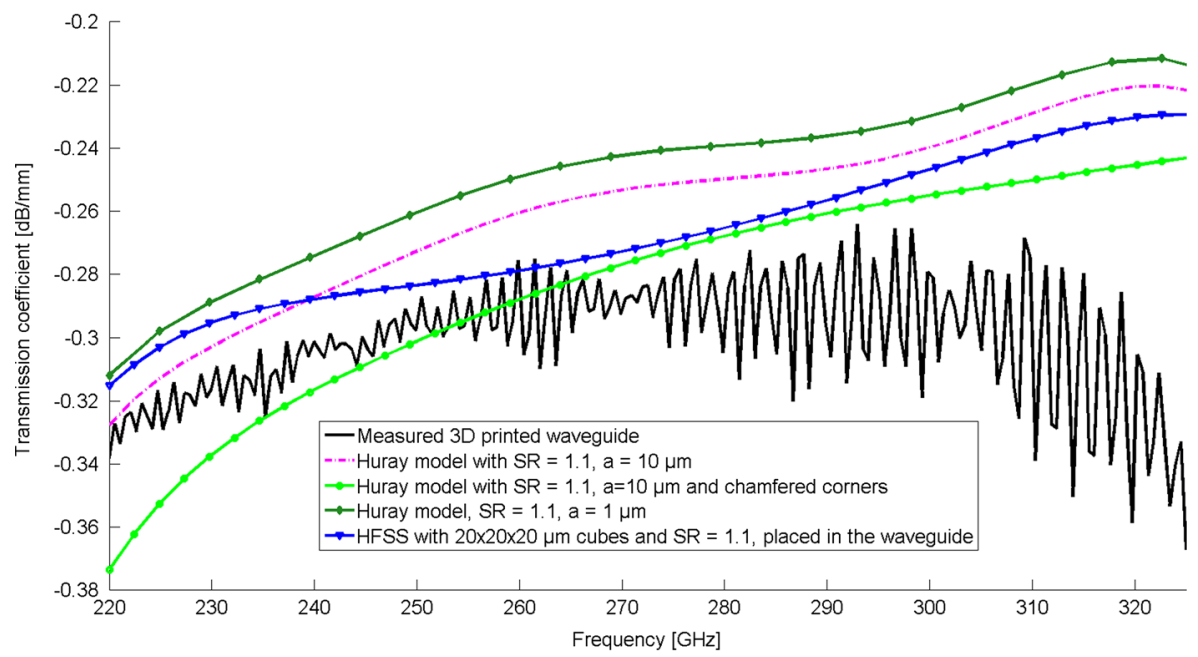

Fig. 8 Transmission coefficient as a function of frequency for the WR3 waveguide. Comparison of 3Dprinted waveguide, Huray model and HFSS simulation. Solid black line: measured 3D-printed waveguide. Pink Huray model with $\mathrm{SR}=1.1, \mathrm{a}=10 \mu \mathrm{m}$. Bright green (more dots along curve): Huray model with $\mathrm{SR}=1.1, \mathrm{a}=10 \mu \mathrm{m}$ and chamfered corners. Dark green (fewer dots along curve): Huray model, $\mathrm{SR}=$ $1.1, \mathrm{a}=1 \mu \mathrm{m}$. Dark blue: HFSS with $20 \times 20 \times 20 \mu \mathrm{m}$ cubes and $\mathrm{SR}=1.1$, placed in the waveguide 
larger but follows the trend of the measured curve reasonably well. Another approach to simulating the surface roughness is to place particles directly in the 3D-modell of the waveguide in Ansoft HFSS. In Fig. 8, the results from simulations with cubic particles of size $20 \times 20 \times 20 \mu \mathrm{m}$ and spacing corresponding to an SR of 1.1 can be seen for the WR3. This approach of simulating the attenuation due to roughness agrees well with the Huray model and thus could be used for investigating other particle sizes, shapes and distributions.

\section{Discussion}

The attenuation for the WR3 band waveguide is of course significantly higher than for WR10. In WR3, the difference between a gold plated commercial waveguide and the 3D-printed is around $0.27 \mathrm{~dB} / \mathrm{mm}$. For WR10, the difference is only $0.045 \mathrm{~dB} / \mathrm{mm}$. The difference between a simulated ideal steel waveguide and the 3Dprinted waveguide is around $0.18 \mathrm{~dB} / \mathrm{mm}$ for the WR3 and around $0.029 \mathrm{~dB} / \mathrm{mm}$ for WR10, thus replacing the steel powder, the manufacturing material in the $3 \mathrm{D}$ printed waveguide with a copper or aluminium powder which can be used in a DMLS process, the losses in the wave guide can be considerably reduced.

The Huray model is found to be rather accurate for the WR10 band which is also consistent with previous findings in [12]. However, when the frequency increases, particularly for the WR3 band, it can be seen that the Huray model, as a function of frequency, does not completely follow the trend of the measurement curve as well as it does for the lower frequency region of the waveguide band. This is probably due to the surface of the WR3 waveguide flanges which are not perfectly flat, creating a small leakage of the RF-power between the waveguide sections particularly visible at the high end of the waveguide band, giving rise to oscillations. Furthermore, the Huray model is based on idealities that assume the same relative spacing between particles on the surfaces of the walls. However, in reality, the largest source of error in the calculations is the accuracy of the spacing of such particles. Also, if a statistical distribution of the sizes of the particles would have been used the end result might have been more accurate. However, in order to acquire a statistical distribution, computerised image analysis of the SEM pictures and also further investigations and calculations are required.

In Figs. 7 and 8, the curves for the Huray model assuming a $1 \mu \mathrm{m}$ diameter for the particles are depicted. It can be seen that the difference between a radius of $10 \mu \mathrm{m}$ and $1 \mu \mathrm{m}$ of the particles with the spacing of particles that was chosen does not significantly affect the attenuation. Thus, the relation of particle size in relation to the wavelength plays a minor role for the Huray model.

The main advantage of using Ansoft HFSS with distributed particles instead of implementing the statistically based Huray model is that the HFSS particle simulation is not limited to small particles and can thus handle larger irregularities in the 3D-printed waveguide which could be of a substantial fraction of the wavelength. However, one large downside with this method is that it is computationally demanding, so it will not work for larger waveguides and/or smaller particles. This is why the method was not used for the WR10 waveguides where the Huray model was in such 
good agreement with the measurements. Different mesh sizes in HFSS were used to investigate the optimal mesh size needed to include the small particles.

Since the smoothness of the waveguide wall is a function of the particle sizes, small particles are, as expected reducing the waveguide loss. However, with a fixed SR of 1.1, the attenuation in the 3D-printed waveguides will not be significantly reduced for e.g. the WR3 band waveguide unless the particle size is decreased below a radius of $1 \mu \mathrm{m}$, according to the Huray model. The chamfered corners have less influence for the WR10-waveguide while they are more of a concern for the WR3.

\section{Conclusion}

Different lengths of WR10 and WR3 waveguides in steel are manufactured with DMLS 3D metal printing. The losses in the waveguides are measured and compared with gold plated commercial waveguides. Comparison to a simulated commercial waveguide in steel is showing an attenuation of 0.029 and $0.18 \mathrm{~dB} / \mathrm{mm}$ for the WR10 and WR 3 respectively. The losses of $0.3 \mathrm{~dB} / \mathrm{mm}$ for WR 3 and $0.05 \mathrm{~dB} / \mathrm{mm}$ for WR10 are of course higher than for a gold-plated CNC-manufactured waveguide but can still be acceptable for some applications and for complicated waveguide structures where other manufacturing methods than 3D-metal printing are not available.

Using optical interferometry measurements and SEM, the stainless steel particle sizes and distribution are analysed. The transmission coefficient due to the surface roughness is evaluated using the Huray model and gives a reasonable accurate value of the waveguide loss as a function of frequency, even though the trend at higher frequencies is less accurate. A more versatile method is implemented where the waveguide loss is simulated using a finite element simulation (particles distributed in the HFSS 3D-model).

Even if DMLS is not providing a lower insertion loss than gold plated CNC machined millimetre and submillimeter waveguides, the fabrication techniques, materials and particle sizes could be further optimized and strongly reduce differences between 3D metal printed waveguides and waveguides realised with other techniques.

Acknowledgements Financial support from the Swedish Foundation for Strategic Research, project "SSF -Development of Processes and Materials in AM" is greatly acknowledged. We acknowledge Mathias Unosson for helpful discussion on 3D-printing and support during manufacturing. We also acknowledge Pedro Berastegui for taking the excellent SEM images of the waveguide. The authors would like to thank the Reviewers for their valuable comments and suggestions.

Open Access This article is distributed under the terms of the Creative Commons Attribution 4.0 International License (http://creativecommons.org/licenses/by/4.0/), which permits unrestricted use, distribution, and reproduction in any medium, provided you give appropriate credit to the original author(s) and the source, provide a link to the Creative Commons license, and indicate if changes were made.

\section{References}

1. B. Zhang, Y.-X. Guo, H. Zirath and Y.P. Zhang, Proc. of the IEEE, 105, 723-736 (2017) 
2. A. D. Squires, E. Constable and R. A. Lewis, J Infrared Millimeter Terahertz Waves, 36, 72-80 (2015)

3. S. Pandey, B. Gupta, and A. Nahata, Optics Express, 21, (2013)

4. D. Headland, W. Withayachumnankul, M. Webb, H. Ebendorff-Heidepriem A. Luiten and D. Abbott, Optics Express, 24, (2016)

5. M. D'Auria, W. J. Otter, J. Hazell, B. T. W. Gillatt, C. Long-Collins, N. M. Ridler, and S. Lucyszyn, IEEE Transactions on Components, Packaging and Manufacturing Technology, 5, 1339-1348 (2015)

6. Muhannad A. Al-Tarifi and Dejan S. Filipovic, "Design and Fabrication of a Full W-Band MultiStep Waveguide 90 Twist", IEEE Microw. and Wireless Compon. Lett, Vol 26, No.11, pp. 903-905 (November 2016)

7. Bing Zhang et al., "Attempt of the Metallic 3D Printing Technology for Millimeter-Wave Antenna Implementations", Microwave Conference (APMC), 2015 Asia-Pacific, IEEE (25 Feb 2016)

8. Ansoft HFSS, http://www.ansys.com/ (2017)

9. OML Inc, https://www.omlinc.com/products/vna-extension-modules (2017)

10. David M.Pozar, "Microwave engineering", 4th edition, ISBN 978-0-470-63155-3, 2012

11. S. Groiss, I. Bardi, O. Biro, K. Preis and K.R. Richter, "Parameters of lossy cavity resonators calculated by the finite element method", IEEE Trans. on Magnetics, vol. 32, no. 3, pp. $894-897$ (1996)

12. Michael B. Griesi, "Characterization of Electrodeposited Copper Foil Surface Roughness for Accurate Conductor Power Loss Modeling", University of south Carolina, 2014

13. P.G. Huray, O. Oluwafemi, J. Loyer, E. Bogatin and X. Ye, "Impact of Copper Surface Texture on Loss: A Model that Works", DesignCon 2010 Proceedings, Santa Clara, CA, 2010

14. E. C. Jordan, Electromagnetic Waves and Radiating Systems (Prentice Hall, 1968)

15. Keysight Technologies, http://www.keysight.com/en/pdx-x201877-pn-N5225A/pna-microwave-networkanalyzer-50-ghz?cc=SE\&lc=eng (2017)

16. OML inc. mm-wave extenders brochure, https://www.omlinc.com/images/pdf/VxxVNA2/OMLVxxVNA2_Brochure.pdf (2017) 